\title{
Application of reduced models for robust control and state estimation of a distributed parameter system
}

\author{
Limei Ding *, Andreas Johansson, Thomas Gustafsson \\ Division of Systems and Interaction, Luleå University of Technology, SE-97187 Luleå, Sweden
}

\section{A R T I C L E I N F O}

Article history:

Received 21 March 2007

Received in revised form 24 April 2008

Accepted 25 April 2008

\section{Keywords:}

Distributed parameter system

Reduced models

Model uncertainty

Paper pulp digester

Robust control

State estimation

\begin{abstract}
A B S T R A C T
This paper studies the application of reduced models of a distributed parameter system for robust process control and state estimation. We take the approach of integrating model reduction, parameter identification, and model uncertainty analysis, in purpose to find an appropriate trade-off between complexity and robust performance. The application example is the temperature system in a continuous paper pulp digester. Physical modeling of this process results in coupled linearized partial differential equations which are then reduced into low-order nominal process models using an orthogonal collocation approximation method.

Two different approaches to obtaining a model uncertainty description are adapted for use on a distributed parameter system with low-order nominal model and shown to produce similar results when tested with measurement data. It is also demonstrated how this uncertainty description, in combination with the reduced model, may be used for robust control design and verification of the control performance on the distributed parameter system.

Finally, the possibility of estimating the distributed process state using a state observer for the reduced process is demonstrated. Measurements of the process state in a certain position is available and is shown to agree with the estimated state at the same position.
\end{abstract}

(c) 2008 Elsevier Ltd. All rights reserved.

\section{Introduction}

The control of distributed parameter systems (DPS) is an important issue in a wide range of the applications, such as spatial profiles, size distributions, fluid flows and material microstructure [1]. First principles modeling of distributed processes lead to nonlinear DPSs of many forms [2], e.g. hyperbolic and parabolic partial differential equations, Navier-Stokes equations, and integro-differential equations. This raises difficulties when it comes to controller synthesis and implementation due to the infinite dimensionality.

In practice, a model approximation is often required in order to produce a low-order model that captures the dominant characteristics of the system $[3,4]$. The topic of model order reduction has been actively discussed in the research field of DPS control. In [5], Li and Christofides applied different model order reduction approaches to a diffusion-convection-reaction process, such as the finite difference method, the Galerkin projection approach, and the orthogonal collocation on finite elements. The reduced models were used in process simulation and control design. Lee et al. [6] presented a general algorithm for robust model reduction of a nonlinear PDE. The study is based on five model order reduction techniques and the authors proposed a resulting model with the lowest

\footnotetext{
* Corresponding author. Tel.: +46 703581716 .

E-mail address: limei.ding@ltu.se (L. Ding).
}

modeling error in the frequency range important for control design.

Also, many first principle models contain unknown parameters, therefore an identification approach for the parameter estimation needs to be considered [7-9]. A low-order model approximation with identified parameters provides a viable solution for obtaining a nominal model suitable for model-based control and monitoring of an industrial DPS. This approach is a grey-box one, because it combines the first principle modeling with data driven parameter identification.

In order to achieve robust stability and performance for the closed-loop, it is necessary to deliver not only a nominal model, but also a reliable estimate of the uncertainty associated with the model. This is one of the main objectives of control-oriented identification which was defined by Helmicki et al. [10] in 1991. Three requirements were set up for control-oriented system identification through the combined use of a system identification method and a robust control design method; (i) the system identification method asymptotically reduces the level of plant uncertainty to the point where a robust controller exists; (ii) the system identification method yields, at each step, an explicit bound on the remaining plant uncertainty associated with the identified nominal model; and (iii) this bound must be in a form compatible with a robust control design method. Many developments and applications of control-oriented identification have been discussed 
since then, e.g. a method that is suitable for linear parameter varying systems [11] and an approach that combines physical modeling and system identification [12].

Control of DPSs is an important application area for control-oriented identification. Model uncertainty cannot be avoided in the reduction of a DPS, thus the robustness must be considered. In 2001, Christofides [13] summarized the methods and applications of robust control to a class of PDE systems.

In this paper, we take the approach of integrating model reduction with parameter identification and model uncertainty analysis, in purpose to find an appropriate trade-off between complexity and robust performance. In particular, the convergence of the identified model parameters with increased model order is analyzed. The model error model approach introduced by Ljung and his coworkers $[14,15]$ is applied to evaluate the uncertainty associated with the nominal model. Another approach to uncertainty modeling is also applied where the nominal process model is compared to the physical process model with parameter values taken from a set of identified values. It is found that the two approaches agree in the most important frequency range.

The low-order nominal process models are then tested in state estimation and robust control. The $H_{\infty}$ loop shaping approach $[16,17]$ makes a trade-off between performance and robustness by synthesizing an $H_{\infty}$ optimal controller with an appropriate robustness margin and a desired open-loop shape. A Kalman filter is suggested to generate process state estimates for the purpose of process monitoring. Although the Kalman filter is built using a loworder model, it is able to estimate the infinite dimensional state of the DPS.

One of the subprocesses studied in [18], a temperature process of the co-current zone in a continuous paper pulp digester at the M-real [19] pulp mill in Husum, Sweden is used as an application example. In the vital steps of the work, i.e. parameter identification and model uncertainty analysis, we use measurement data from this plant. Measurement data are also used for validating the state estimates obtained with the reduced models. For verifying control performance, a frequency domain solution of the original PDE is used to simulate the process.

The outline of the paper is given below: Next section introduces a simplified linear physical model of a process with transport phenomena. Both an infinite dimensional model and finite dimensional approximations of the model are discussed, then parameter identification of this process is studied. In Section 3, the errors of the reduced models are estimated and upper bounds of the errors are defined. The basic principle of loop-shaping is briefly presented in Section 4, then robust control and state estimation using the reduced models are demonstrated. Finally, the research work is concluded in Section 5.

\section{A physical model and the model approximation}

Conservation laws of mass, momentum and energy form the basis of the field of transport phenomena. These laws applied to the flow of fluids result in the partial differential equations which describe the dynamics of variables of a system, such as temperatures and concentrations, with respect to time and position. A vertical tubular reactor is an example of such processes with transport phenomena.

\subsection{Control of a DPS and its implementation problem}

A vertical tubular reactor of co-current flow in two phases, liquid phase and solid phase, is a DPS and can be modeled by PDEs based on conservation laws. A direct analytical solution of the control synthesis problem for a DPS modeled by PDEs often results in a controller that requires distributed measurements and actuators. In practice such a controller is not implementable. On the left side of Fig. 1, a vertical tubular reactor in common uses is illustrated. There are a finite set of the measurements and actuators, two inputs and two outputs. Satisfactory control of the process must be achieved using this finite set of measurements and actuators. A finite dimensional control synthesis can be made based on a reduced process model, which describes the process dynamics at a finite set of positions, the boundary points $z_{0}$ and $z_{N+1}$, and $N$ interior points $z_{1}, z_{2}, \ldots, z_{N}$.

On the right side of Fig. 1, a specified process is shown and the process has the main features of a vertical reactor. This is the cocurrent zone in a continuous paper pulp digester and a detailed introduction of the digester can be found in [18]. The top position of this process is denoted as $z_{0}$, the upper extraction at the bottom position is denoted as $z_{\text {ext }}$ and the positions of the three measurements are $z_{0}, z_{\mathrm{c} 6}$ and $z_{\text {ext }}$. The dynamics of the flow temperatures in the co-current zone of a digester can be modeled using a simplified physical model consisting of coupled PDEs $[20,18]$ :

$\frac{\partial T_{\mathrm{f}}(z, t)}{\partial t}=-v_{\mathrm{f}} \frac{\partial T_{\mathrm{f}}(z, t)}{\partial z}+d_{\mathrm{f}}\left(T_{\mathrm{c}}(z, t)-T_{\mathrm{f}}(z, t)\right)$
$\frac{\partial T_{\mathrm{c}}(z, t)}{\partial t}=-v_{\mathrm{c}} \frac{\partial T_{\mathrm{c}}(z, t)}{\partial z}+d_{\mathrm{c}}\left(T_{\mathrm{f}}(z, t)-T_{\mathrm{c}}(z, t)\right)$

where $T_{\mathrm{f}}(z, t)$ and $T_{\mathrm{c}}(z, t)$ are the temperatures of the liquid phase and the solid phase, for the specified process in a digester, free liquor and wood chips; the model parameters $v_{\mathrm{f}}$ and $v_{\mathrm{c}}$ deal with the velocities of the liquid flow and solid flow; and the two additional model parameters $d_{\mathrm{f}}$ and $d_{\mathrm{c}}$ are related to the interphase diffusion of the two flows. The independent variables $z$ and $t$ are the vertical position of the reactor and the time, respectively. The vertical length is normalized, so we define that the top position is $z_{0}=0$ and the bottom position is $z_{\mathrm{ext}}=1$.

The steam temperature at the top of the digester is selected as a manipulated variable, and the temperatures of the both flows at the top position are assumed to be the same as the steam temperature, i.e. $T_{\mathrm{f}}\left(z_{0}, t\right)=T_{\mathrm{c}}\left(z_{0}, t\right)=T_{\mathrm{s}}\left(z_{0}, t\right)$. The temperature at the upper extraction is chosen as the output of the process. Thus the input and the output are defined as

$u(t)=T_{\mathrm{s}}\left(z_{0}, t\right), \quad y(t)=T_{\mathrm{f}}\left(z_{\mathrm{ext}}, t\right)$

\subsection{Model solution in frequency domain}

Eqs. (1a) and (1b) formulate a time domain model of the process. The frequency domain model, i.e. the process transfer functions, can be found directly from (1a) and (1b), and is given as

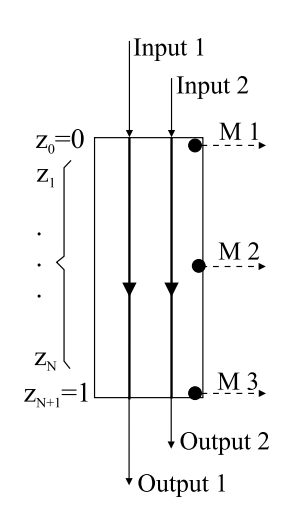

A vertical reactor

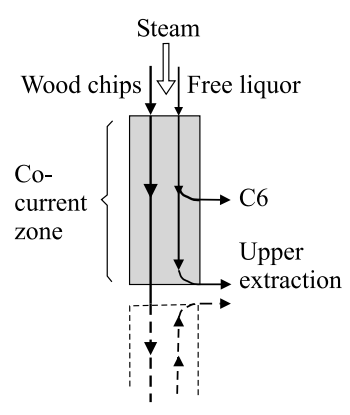

The co-current zone in a continuous digester
Fig. 1. Finite set of the measurements and actuators in vertical reactors. 
$T_{\mathrm{f}}(z, s)=G_{1}(z, s) T_{s}(s)$

$T_{\mathrm{c}}(z, s)=G_{2}(z, s) T_{\mathrm{s}}(s)$

where the infinite dimensional transfer functions $G_{1}(z, s)$ and $G_{2}(z, s)$ are

$$
\begin{aligned}
G_{1}(z, s)= & \frac{\beta_{1}-\left(\lambda_{2}(s)+\alpha_{1}(s)\right)}{\lambda_{1}(s)-\lambda_{2}(s)} \mathrm{e}^{\lambda_{1}(s) z} \\
& +\frac{\left(\lambda_{1}(s)+\alpha_{1}(s)\right)-\beta_{1}}{\lambda_{1}(s)-\lambda_{2}(s)} \mathrm{e}^{\lambda_{2}(s) z} \\
G_{2}(z, s)= & \frac{\beta_{2}-\left(\lambda_{2}(s)+\alpha_{2}(s)\right)}{\lambda_{1}(s)-\lambda_{2}(s)} \mathrm{e}^{\lambda_{1}(s) z} \\
& +\frac{\left(\lambda_{1}(s)+\alpha_{2}(s)-\beta_{2}\right.}{\lambda_{1}(s)-\lambda_{2}(s)} \mathrm{e}^{\lambda_{2}(s) z}
\end{aligned}
$$

where

$\begin{array}{ll}\alpha_{1}(s)=\frac{s+d_{\mathrm{f}}}{v_{\mathrm{f}}}, & \beta_{1}=\frac{d_{\mathrm{f}}}{v_{\mathrm{f}}} \\ \alpha_{2}(s)=\frac{s+d_{\mathrm{c}}}{v_{\mathrm{c}}}, & \beta_{2}=\frac{d_{\mathrm{c}}}{v_{\mathrm{c}}}\end{array}$

and

$$
\begin{aligned}
\lambda_{1,2}(s)= & \frac{1}{2}\left(-\left(\alpha_{1}(s)+\alpha_{2}(s)\right)\right) \\
& \pm \frac{1}{2} \sqrt{\left(\alpha_{1}(s)+\alpha_{2}(s)\right)^{2}-4\left(\alpha_{1}(s) \alpha_{2}(s)-\beta_{1} \beta_{2}\right)}
\end{aligned}
$$

The derivation of the transfer function can be referred to [20].

\subsection{Model reduction and model parameter estimation}

To deal with the infinite dimensionality of the process, a model reduction method must be applied, by which an infinite dimensional model can be reduced to a low-order (finite dimensional) approximation.
In our previous research [18], the OC approximation of the PDEs (1a) and (1b) is provided. The reduced state space model can be expressed in the form

$\dot{x}(t)=A x(t)+B u(t)$

$y(t)=C x(t)+D u(t)$

The state vector of the low-order model is $x=\left[x_{f}^{\mathrm{T}} x_{\mathrm{c}}^{\mathrm{T}}\right]^{\mathrm{T}}$ where

$x_{\mathrm{f}}^{\mathrm{T}}(t)=\left[\begin{array}{llll}T_{\mathrm{f}}\left(z_{1}, t\right) & T_{\mathrm{f}}\left(z_{2}, t\right) & \cdots & T_{\mathrm{f}}\left(z_{N+1}, t\right)\end{array}\right]$

$x_{\mathrm{c}}^{\mathrm{T}}(t)=\left[\begin{array}{llll}T_{\mathrm{c}}\left(z_{1}, t\right) & T_{\mathrm{c}}\left(z_{2}, t\right) & \cdots & T_{\mathrm{c}}\left(z_{N+1}, t\right)\end{array}\right]$

i.e. the temperatures in the collocation points $z=z_{0}, z_{1}, \ldots, z_{N+1}$, and $N$ is defined as the number of interior collocation points. The matrices $A, B, C$ and $D$ are

$A=\left[\begin{array}{cc}-v_{\mathrm{f}} A_{1}-d_{\mathrm{f}} I & d_{\mathrm{f}} I \\ d_{\mathrm{c}} I & -v_{\mathrm{c}} A_{1}-d_{\mathrm{c}} I\end{array}\right]$

$B=\left[\begin{array}{l}-v_{\mathrm{f}} B_{1} \\ -v_{\mathrm{c}} B_{1}\end{array}\right]$

$C=\left[\begin{array}{ll}C_{1} & 0\end{array}\right]$

$D=0$

and

$A_{1}=\left[\begin{array}{ccc}\left.\frac{\partial l_{1}}{\partial z}\right|_{z=z_{1}} & \cdots & \left.\frac{\partial l_{N+1}}{\partial z}\right|_{z=z_{1}} \\ \vdots & \vdots & \vdots \\ \left.\frac{\partial l_{1}}{\partial z}\right|_{z=z_{N+1}} & \cdots & \left.\frac{\partial l_{N+1}}{\partial z}\right|_{z=z_{N+1}}\end{array}\right]$

$B_{1}=\left[\begin{array}{lll}\left.\frac{\partial l_{0}}{\partial z}\right|_{z=z_{1}} & \cdots & \left.\frac{\partial l_{0}}{\partial z}\right|_{z=z_{N+1}}\end{array}\right]^{T}$

$C_{1}=\left[\begin{array}{lll}l_{1}\left(z_{N+1}\right) & \cdots & l_{N+1}\left(z_{N+1}\right)\end{array}\right]$

where the functions $l_{j}(z)$ are Lagrange interpolation polynomials with the interpolation points chosen as the interior collocation points $z_{1}, z_{2}, \ldots, z_{N}$, and the boundary points $z_{0}=0$ and $z_{N+1}=1$.
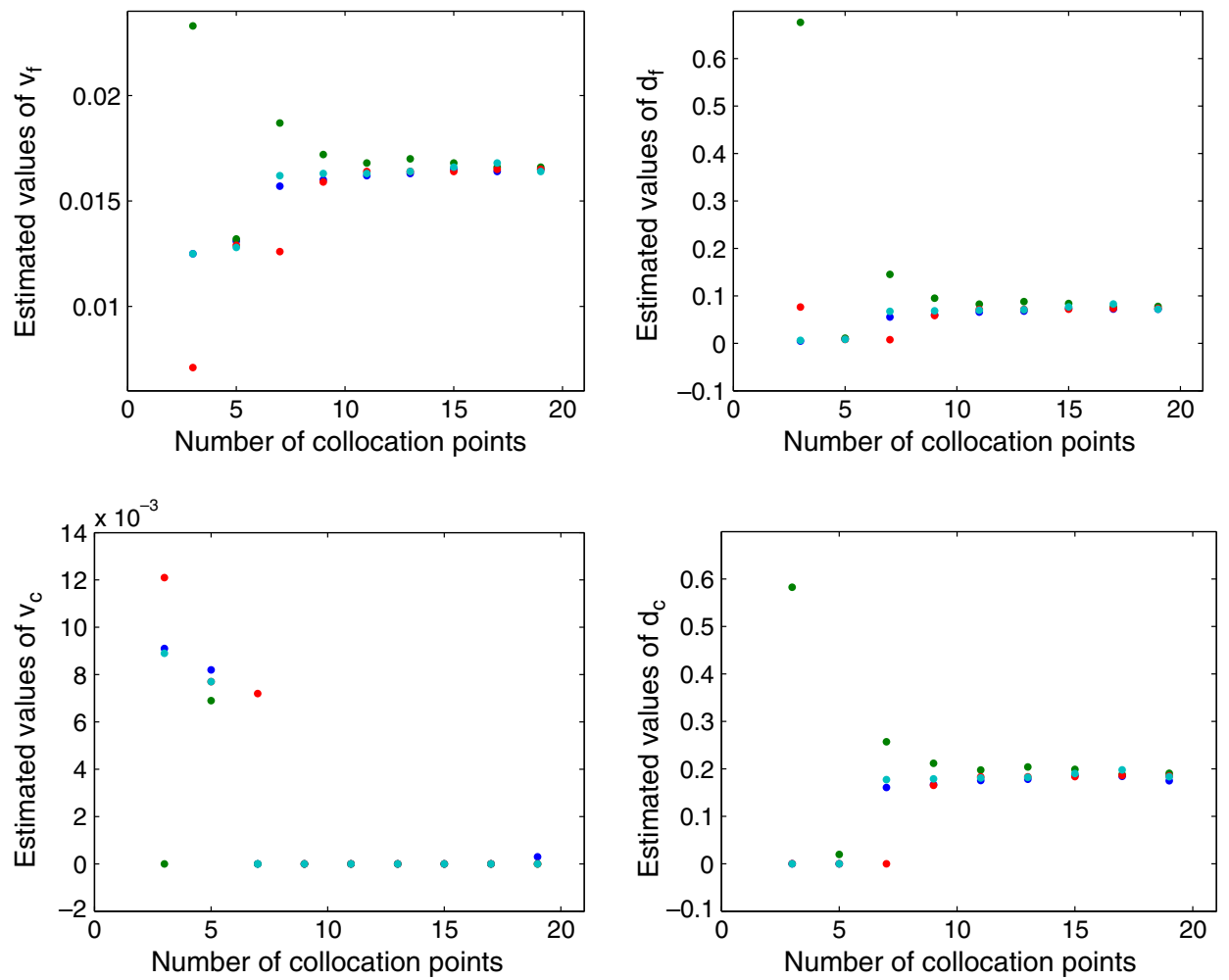

Fig. 2. Unknown parameter estimates as a function of the number of collocation points $N$. 
Table 1

Parameter intervals

\begin{tabular}{ll}
\hline Parameter & Interval \\
\hline$v_{\mathrm{f}}$ & $V_{\mathrm{f}}=[0.0162,0.0170]$ \\
$d_{\mathrm{f}}$ & $D_{\mathrm{f}}=[0.0659,0.0880]$ \\
$v_{\mathrm{c}}$ & $V_{\mathrm{c}}=[0.0000,0.0003]$ \\
$d_{\mathrm{c}}$ & $D_{\mathrm{c}}=[0.1748,0.2040]$ \\
\hline
\end{tabular}

The identity matrix "I" and the zero matrix " 0 " that appear in the matrices $A$ and $C$ have the same dimensions as matrices $A_{1}$ and $C_{1}$, respectively. For details of the state space model, please refer to [18].

For an arbitrary position $z$, the following approximation is available [18]:

$T_{\mathrm{f}}(z, t)=\sum_{j=1}^{N+1} T_{\mathrm{f}}\left(z_{j}, t\right) l_{j}(z)+T_{\mathrm{s}}(t) l_{0}(z)$

$T_{\mathrm{c}}(z, t)=\sum_{j=1}^{N+1} T_{\mathrm{c}}\left(z_{j}, t\right) l_{j}(z)+T_{\mathrm{s}}(t) l_{0}(z)$

The parameter vector of the reduced model is

$\theta=\left[\begin{array}{llll}v_{\mathrm{f}} & d_{\mathrm{f}} & v_{\mathrm{c}} & d_{\mathrm{c}}\end{array}\right]^{\mathrm{T}}$

and it is the same as that can be defined for the infinite dimensional physical model (1a) and (1b). In [18] the four unknown parameters shown in (17) are estimated using the least squares method. The reduced models with $N=3,5,7,9$ are selected for the parameter identification, and the data used for both parameter identification and model validation are measurement data from the pulp digester at the M-real paper plant in Husum, Sweden.

In the current research we extend the identification work by selecting more of the reduced models with different choice of $N$. It appears that the parameter values converge to small intervals when the number of collocation points $N$ increases. Fig. 2 shows the parameter values as functions of $N$ for a number of identification experiments. It can be seen from Fig. 2 that for $N \geqslant 11$, the parameter values are confined to small intervals, and those intervals are specified in Table 1 . The OC approximations with different selection of $N$ provide a set of nominal models for the model applications studied later in this paper.

\section{Model error analysis}

We assume that the "true" process belongs to the set $\Pi$ of processes $G_{p}$, where $G_{p}$ denotes a perturbed process. Robustness of a controller then implies that it satisfies its specification for all $G_{\mathrm{p}} \in \Pi$. Process perturbations can be constructed in different ways [17], such as additive uncertainty, multiplicative uncertainty, normalized-coprime-factor uncertainty, etc. Choosing the additive uncertainty for our discussion, $\Pi$ consists of all $G_{\mathrm{p}}$ on the form

$G_{\mathrm{p}}(s)=\widehat{G}(s)+W_{\mathrm{A}}(s) \Delta_{\mathrm{A}}(s)$

where $\widehat{G}(s)$ is a nominal process model, $W_{\mathrm{A}}(s)$ is an uncertainty weight, and $\Delta_{\mathrm{A}}(s)$ is an arbitrary complex function satisfying $\left|\Delta_{\mathrm{A}}(i \omega)\right|<1$ for all frequencies, and the subscript $\mathrm{A}$ in $W_{\mathrm{A}}$ and $\Delta_{\mathrm{A}}$ denotes additive uncertainty.

In the preceding section, a set of nominal models were obtained by taking the OC approximations of the PDEs (1a) and (1b), and using the parameter estimates shown in Fig. 2. A satisfactory robust controller synthesized from such a nominal model must be able to tolerate the model perturbations due to model simplification, model order reduction and the parameter estimation. Thus it is interesting to analyze the perturbations in the frequency domain.
3.1. Model errors caused by parameter perturbation and model order reduction

One may separately discuss the model perturbations due to different reasons. In this subsection we discuss the model errors caused by parameter perturbation and model order reduction. We assume that a subset $\Pi_{1} \subset \Pi$ consists of the PDE transfer function (3) and (4) with different parameter values. Using the parameter value intervals specified in Table 1 , an upper bound $l_{\mathrm{A}}(w)$ for the magnitude $\left|W_{\mathrm{A}}(i \omega)\right|$ of the uncertainty weight can then be obtained as [17]:

$l_{\mathrm{A}}(\omega)=\max _{\theta \in \Theta}\left|G_{1}(1, \mathrm{i} \omega)-\widehat{G}(\mathrm{i} \omega)\right|$

where $\theta=\left(v_{\mathrm{f}}, d_{\mathrm{f}}, v_{\mathrm{c}}, d_{\mathrm{c}}\right)$ and $\Theta=V_{\mathrm{f}} \times D_{\mathrm{f}} \times V_{\mathrm{c}} \times D_{\mathrm{c}}$ is the Cartesian product of the intervals in Table 1 .

As nominal model $\widehat{G}(s)$, the identified low-order model (11) with $N=7$ and parameter values $v_{\mathrm{f}}=0.0161, d_{\mathrm{f}}=0.0476$, $v_{\mathrm{c}}=0.0078, d_{\mathrm{c}}=0.0420$ is selected. Note that these parameter values are valid only for this particular low-order model. They do not belong to the parameter intervals of Table 1 since they are not necessarily related to the true parameter values of the process.

The upper plot in Fig. 3 shows the amplitude of the frequency response of the nominal model and the PDE model (3) and (4) with parameter values selected from the intervals in Table 1 while the lower plot shows the amplitude of the difference $\left|G_{1}(1, \mathrm{i} \omega)-\widehat{G}(\mathrm{i} \omega)\right|$. The final step in selecting the uncertainty weight is to find a simple low-order weight $W_{\mathrm{A} 1}(s)$ that satisfies $\left|W_{\mathrm{A} 1}(i \omega)\right| \geqslant l_{\mathrm{A}}(\omega)$ for all $\omega \geqslant 0$. Our choice

$W_{\mathrm{A} 1}(s)=12 \frac{s(s / 2+1)}{(s / 0.06+1) 2}$

is shown in the figure with a dot-dashed line.

Because $l_{\mathrm{A}}(\omega)$ defined in (19) is obtained by taking the difference between the PDE model that is perturbed by the uncertain parameters and the reduced model, it is clear that the weight function (20) gives an upper bound of the model error due to the parameter perturbation and the model order reduction. However this error bound does not include the model errors caused by model simplification, etc. The weight function $W_{\mathrm{A} 1}$ thus only partly represents the model errors of a reduced OC model.
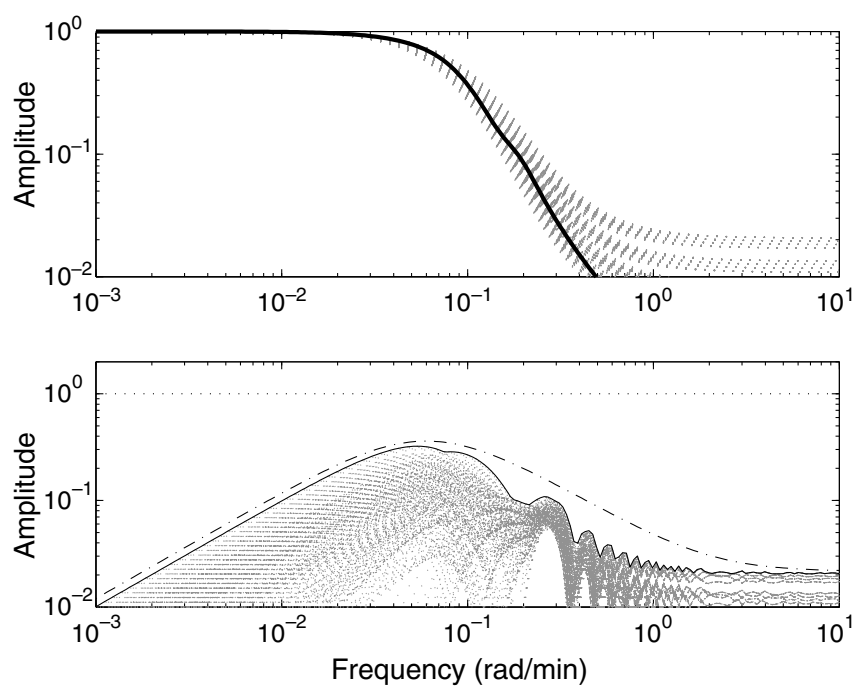

Fig. 3. Upper: PDE model (3) and (4) with parameter values from Table 1 (grey dashed) and nominal model (black, solid). Lower: Difference between PDE model and nominal model (grey, dashed) with upper bound $l_{\mathrm{A}}$ (black, solid) and selected uncertainty weight $W_{\mathrm{A} 1}$ (black, dot-dashed). 


\subsection{Overall model errors}

To analyze the overall errors of a reduced model, one should examine the difference between the simulation data using the reduced model and measurement data collected from the process, since this difference represents the model errors caused by all different reasons.

An approach proposed by Ljung [14] that deals with model error models is an alternative for the model error estimation. We express a real plant as follows:

$y=G u+v$

where $y$ is the observed output variable of the plant, $G$ is the true process and $v$ is the system noise. A model residual $\varepsilon$ can be computed

$\varepsilon=y-\widehat{G} u$

where $\widehat{G}$ is a nominal model that is estimated from the data $y$ and $u$. The model residual $\varepsilon$ can be separated into model error $\Delta$ and disturbances $w$ [14]:

$\varepsilon=\Delta u+w$

where no further requirement is put on $w$.

Fig. 4 shows the basic idea of model error modeling [14,15]. The upper plot illustrates the nominal model $\widehat{G}$ along with the uncertainty region (dashed lines) and the center of the uncertainty region, $\widehat{G}+\Delta$. The lower plot demonstrates the model error model $\Delta$ and its uncertainty band (dashed lines). This uncertainty contains zero except for an interval at high frequencies, where the nominal model is outside the so constructed uncertainty region shown in the upper plot.

A nominal model could fail in a traditional correlation test, but pass a control-oriented model validation test if the norms of its model error model $\Delta$ and disturbance $w$ are limited in an acceptable level [14]. An advantage with the model error model concept is that the nominal model still can be used for control design, even if it is falsified by $\Delta$. This is the case, for example, if the falsification takes place in "unimportant" frequency regions [15].

A black-box identification procedure named prediction error method [21,22] is employed to estimate the model error models of the nominal models. The model error model of the OC nominal model with $N=7$ is estimated and the estimate passes the correlation test. In Fig. 5, the estimated model error model is plotted using a solid line, and its $99 \%$ confidence interval is plotted using dotted lines.
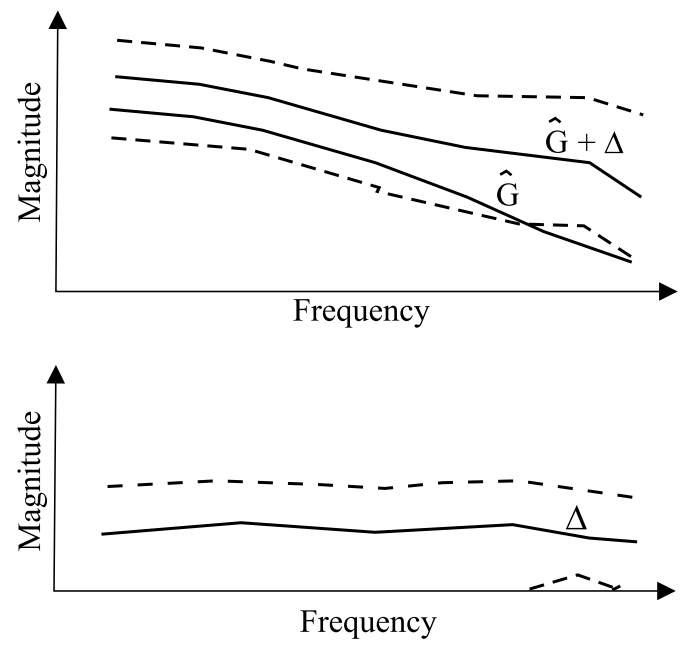

Fig. 4. The basic idea of model error modeling.

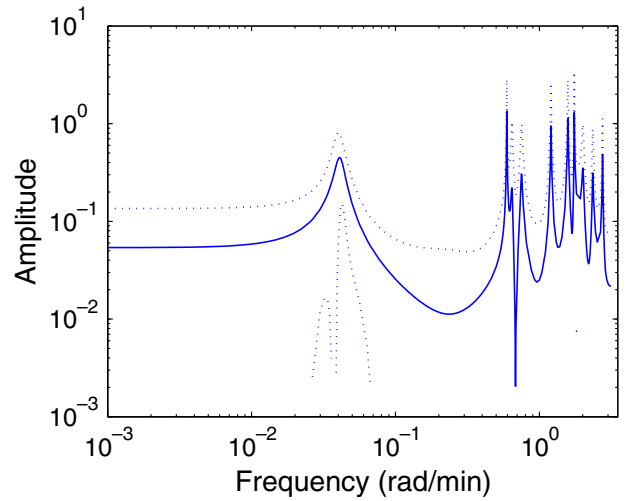

Fig. 5. The model error model estimated for the OC model with $N=7$.

Putting the representations of $\varepsilon$ given by (22) and (23) together, we have

$y=\widehat{G} u+\Delta u+w$

This equation suggests an additive uncertainty $\Delta$, and $\widehat{G}+\Delta$ represents a perturbed process. We adopt the model error model to represent the perturbed process as follows:

$G_{\mathrm{p}}(s)=\widehat{G}(s)+\Delta(s)$

where the model error model $\Delta(s)$ is assumed to be confined within the $99 \%$ confidence interval. For the control synthesis problem using the perturbed model in (25), we assume that the "true plant" is some $G_{\mathrm{p}}(s) \in \Pi$.

\subsection{Comparison}

Ljung et al. [21,15,23] analyzed the error in standard identification problems. The error can be divided into a bias, due to system dynamics which is not captured by the estimated nominal model, and a variance, due to noise affecting the data. Although we obtained the model in this study by applying a grey-box approach, the main model error still consists of the two parts mentioned above. The model simplification and the model order reduction definitely lead to model structure error, i.e., the bias. The variance of parameter estimation also exists. Since we use actual measurement data in our study, the properties of the disturbances may be more complicated than that assumed for the standard identification problems, since there are feedback phenomena involved in the process due to the liquor circulation, and that the measurement noise is not guaranteed to be white. Additional errors may appear due to these factors.

In Fig. 6, the model error model of the nominal OC model with $N=7$ and the weight function defined by (20) are plotted together. We approximately divide the frequency range into three subintervals: a low-frequency region (L-region), a mid-frequency region (M-region) and a high-frequency region (H-region). It is obvious that the estimated model error model is almost covered by $W_{\mathrm{A} 1}$ in the M-region, while it exceeds $W_{\mathrm{A} 1}(s)$ in the L-region and the $\mathrm{H}$-region. Hence the weight function $W_{\mathrm{A} 1}$ is an acceptable model error description in the M-region.

The upper plots of Fig. 3 demonstrate the frequency properties of the infinite dimensional model and a nominal model. The properties in a frequency range approximately corresponding the M-region are characteristic for the process, therefore the M-region is an important frequency range.

The agreement between the different model error estimates in the M-region shown in Fig. 6 supports the legitimacy of the simplified physical model (1a) and (1b), and this implies that the errors 


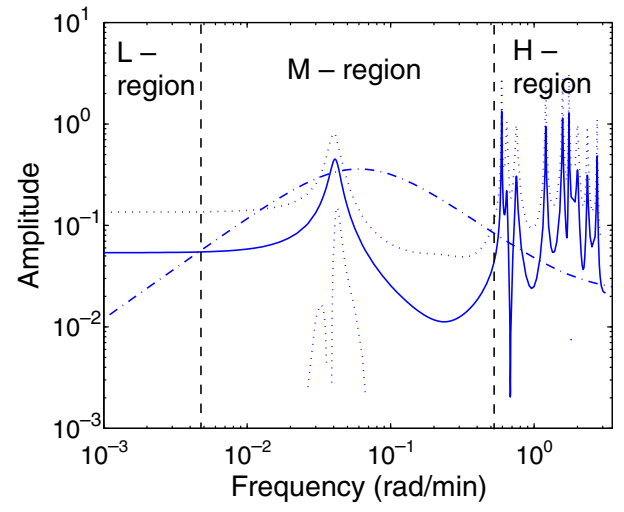

Fig. 6. Comparing $W_{\mathrm{A} 1}(s)$ to the model error model of the reduced OC model with $N=7$. Solid line: estimate of the model error model; dotted lines: $99 \%$ confidence interval of the model error model; dot-dashed line: weight function $W_{\mathrm{A} 1}(s)$.

of the reduced models in this frequency region are caused mainly by the parameter perturbation and the model order reduction. We have estimated model error models for the OC models with $N=3,5, \ldots, 17,19$, then compared to $W_{\mathrm{A} 1}$. The results are similar as those for $N=7$.

The model error model can also be used for assessing the principal deficiencies of the physical model and improve it. Fig. 6 shows that the main differences between the model error model and $W_{\mathrm{A} 1}(s)$ appear particularly in the L-region and the H-region. The difference in the L-region may be due to the neglecting of some energy terms present in the fundamental model in [24]. Because of the model simplification, the following terms are neglected: temperature change due to mass variations, temperature change due to external stream, energy transfer due to diffusion, and temperature change due to heat released by reactions. An improvement of the simplified physical model ( $1 \mathrm{a}$ ) and ( $1 \mathrm{~b}$ ) can be made by adding a term that is proportional to the temperature in the equations. Fig. 7 demonstrates the model errors obtained in the same way as described for Fig. 3 when adding a term $-k_{\mathrm{f}} T_{\mathrm{f}}$ to the right hand side of (1a) and letting $k_{\mathrm{f}} \in[0,0.001]$. This improvement obviously reduces the difference between $W_{\mathrm{A} 1}$ and the model error model estimates in the L-region.

In order to find a weight function $W_{\mathrm{A}}(s)$ that covers the overall errors of a nominal model to ensure controller robustness, we state that:
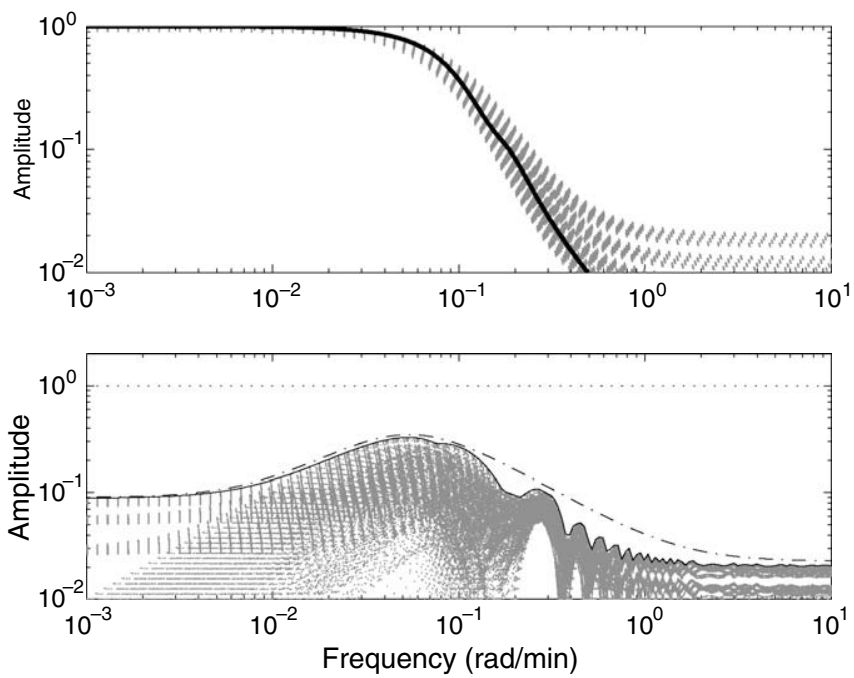

Fig. 7. Improvement of weight function $W_{\mathrm{A} 1}(s)$ (dot-dashed line) by adding $-k_{\mathrm{f}} T_{\mathrm{f}}$ in the model.
The amplitude of $W_{\mathrm{A}}(s)$ should be greater than the upper bound of the $99 \%$ confidence interval of the model error model estimate.

The upper plot in Fig. 8 illustrates the model error model (solid line) of the $\mathrm{OC}$ nominal model with $N=7$, and a weight function (dashed line) that covers the overall errors.

In the H-region, the large amplitude values of the model error model estimate is most likely caused by the measurement noise, since the amplitude of the process must be small in this frequency region due to the process physics. It is unnecessary to take $\left|W_{\mathrm{A}}(i \omega)\right|$ greater than the $99 \%$ confidence interval of the model error model estimate in the H-region, while it is still reasonable to have it greater than $\left|W_{\mathrm{A} 1}(i \omega)\right|$. The dashed weight function $W_{\mathrm{A}}(s)$ shown in the upper plot of Fig. 8 is thus actually a conservative selection.

Based on the analysis presented above, a weight function $W_{\mathrm{A}}(s)$ that covers the overall model errors of a nominal model is defined as follows:

In the $L$ - and M-region, the amplitude of $W_{\mathrm{A}}(s)$ is defined to be greater than the upper bound of the $99 \%$ confidence interval of the model error model estimate.

In the H-region, the amplitude of $W_{\mathrm{A}}(s)$ is defined to be greater than $W_{\mathrm{A} 1}(i \omega)$.

An improved weight function is shown with the dot-dashed line in the upper plot of Fig. 8, and the function is

$W_{\mathrm{A}}(s)=\frac{0.06(s+0.0135)\left(s^{2}+2 s+0.3\right)}{(s+0.81)\left(s^{2}+0.0267 s+0.002\right)}$

The lower plot of Fig. 8 illustrates weight functions selected for the OC nominal models with $N=3,5,7,17$, respectively, which can be used to analyze control system robustness. In general, it can be seen from the figure that higher order models result in smaller error bounds.
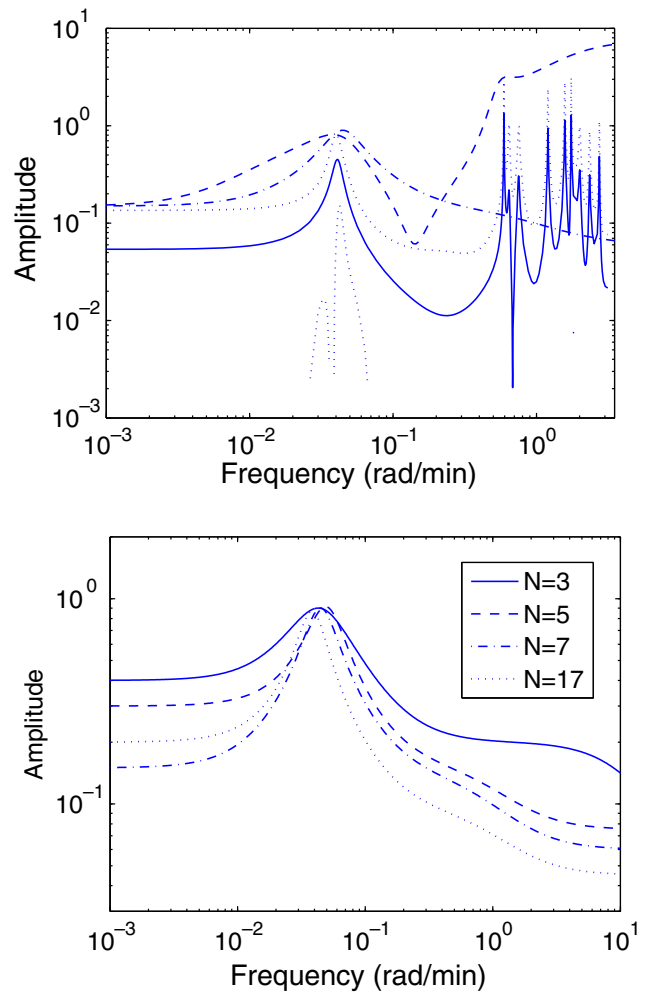

Fig. 8. Upper: weight function (dashed line) of the overall errors defined based on the estimate of the model error models of the OC nominal model with $N=7$, and an improved weight function $W_{\mathrm{A}}(s)$ (dot-dashed line); lower: weight functions of the model errors of the OC nominal models in different orders. 
The discussion of the model errors estimated in different ways is helpful to get better understanding of the error sources, and in the future this may help to improve a reduced model by minimizing model errors caused by different reasons. For example, to minimize the errors in M-region by improving the order reduction methods, to minimize the errors in L-region by re-including some terms in the physical process model that were removed when simplifying it.

\section{Application of the reduced models on process control and state estimation}

In this section, the reduced models are used for controller synthesis and design of a Kalman filter. The model quality and applicability can be evaluated by direct applications of the reduced models in process control and state estimation. The control robustness and the residuals of state estimation will be analyzed.

\subsection{Process robust control}

The estimated model error models provide a description of the model errors in the frequency domain which is helpful for robust controller synthesis.

Fig. 9 illustrates a closed-loop control system for a perturbed process with an additive uncertainty, where $\Delta_{\mathrm{A}} W_{\mathrm{A}}=\Delta$ and the perturbed process $\widehat{G}+\Delta$ is the same as that represented by (25). The weight function $W_{\mathrm{A}}$ gives an upper bound to the additive uncertainty and $\Delta_{\mathrm{A}}$ represents a normalized dynamics that satisfies $\left\|\Delta_{\mathrm{A}}\right\|_{\infty} \leqslant 1$.

Robust control synthesis based on the $H_{\infty}$ loop shaping procedure computes a stabilizing $H_{\infty}$ controller $K$ for the plant $\widehat{G}$ to shape the singular value plot of the loop transfer function $\widehat{G} K$. Roughly speaking, the function $\widehat{G} K$ is shaped to have desired loop shape $G_{d}$ with accuracy $\gamma$ [25], then,

$\underline{\sigma}(\widehat{G}(\mathrm{j} \omega) K(\mathrm{j} \omega)) \geqslant \frac{1}{\gamma} \bar{\sigma}\left(G_{d}(\mathrm{j} \omega)\right)$ for all $\omega<\omega_{0}$

$\bar{\sigma}(\widehat{G}(\mathrm{j} \omega) K(\mathrm{j} \omega)) \leqslant \gamma \underline{\sigma}\left(G_{d}(\mathrm{j} \omega)\right)$ for all $\omega>\omega_{0}$

where $\omega_{0}$ is the $0 \mathrm{db}$ crossover frequency of the singular value plot of $G_{d}(\mathrm{j} \omega)$.

The accuracy $\gamma$ is defined as $[25,26]$ :

$\gamma=\min _{K}\left\|\left[\begin{array}{c}I \\ K\end{array}\right](I+G K)^{-1}[G I]\right\|_{\infty}$

Robustness margin of a closed-loop system controlled by the robust controller is defined as $\epsilon=\gamma^{-1}$. Obviously, a small value of $\gamma$ implies that the controlled system has a large robustness margin [26].

Applying the robust control approach from [17,16,26], a closedloop control system of a perturbed process can be illustrated using a general control configuration consisting of a generalized plant, a controller $K$ and a normalized uncertainty, in our case $\Delta_{\mathrm{A}}$. From Fig. 9 , the generalized plant is obtained as

$$
\left[\begin{array}{c}
y_{\Delta} \\
z \\
\hline e
\end{array}\right]=\left[\begin{array}{ll|l}
0 & 0 & W_{A} \\
W_{p} & W_{p} & W_{p} \hat{G} \\
\hline-1 & -1 & \hat{G}
\end{array}\right]\left[\begin{array}{l}
u_{\Delta} \\
w \\
\hline u
\end{array}\right]
$$

where $u_{\Delta}=\left(\Delta_{\mathrm{A}}\right) y_{\Delta}$, and assuming $r=0$ then $e=-y$ and $u=-K y$.

An $N \Delta$ structure $[17,16,26]$ of the closed-loop system is then given as

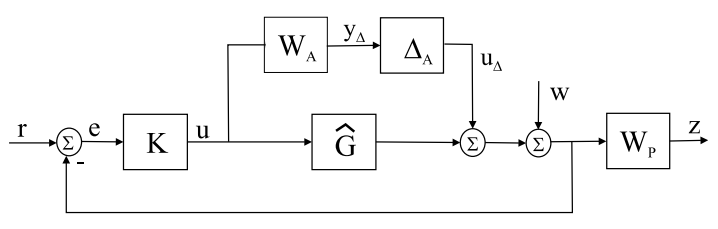

Fig. 9. A closed-loop robust control of a perturbed process with additive uncertainty.

$$
\left[\begin{array}{c}
y_{\Delta} \\
z
\end{array}\right]=\left[\begin{array}{ll}
N_{11} & N_{12} \\
N_{21} & N_{22}
\end{array}\right]\left[\begin{array}{l}
u_{\Delta} \\
w
\end{array}\right]
$$

where $N_{11}=N_{12}=-W_{\mathrm{A}} K S, N_{21}=N_{22}=W_{\mathrm{p}} S$, and $S=(I+\widehat{G} K)^{-1}$ is the system sensitivity function.

Using the $N \Delta$ structure, the following criterion [17] must hold for robust stability of the closed-loop system:

Assume that the system is nominally stable, $\Delta_{\mathrm{A}}$ is a set of stable full complex matrices, and $\left\|\Delta_{\mathrm{A}}\right\|_{\infty} \leqslant 1$, then the perturbed system is robustly stable if and only if

$\bar{\sigma}\left(N_{11}(\mathrm{i} \omega)\right)<1 \quad \forall \omega$

The closed-loop system illustrated by Fig. 9 is thus robustly stable if and only if

$\bar{\sigma}(K(\mathrm{i} \omega) S(\mathrm{i} \omega))<\left|W_{\mathrm{A}}(\mathrm{i} \omega)\right|^{-1} \quad \forall \omega$

or equivalently

$\bar{\sigma}(T(\mathrm{i} \omega))<|\widehat{G}(\mathrm{i} \omega)|\left|W_{\mathrm{A}}(\mathrm{i} \omega)\right|^{-1} \quad \forall \omega$

where $T(\mathrm{i} \omega)=\widehat{G}(\mathrm{i} \omega) K(\mathrm{i} \omega)(I+\widehat{G}(\mathrm{i} \omega) K(\mathrm{i} \omega))^{-1}$.

The robust stability criterion (34) will be utilized for an application example of robust process control discussed in the next subsection.

\subsection{Digester temperature control based on the $H_{\infty}$ loop shaping procedure}

For process control one prefers to use a low-order model. In this study we choose nominal models with different $N$ in control synthesis for comparing the system robust stability and performance.

In order to achieve good disturbance rejection and command tracking, we want to synthesize a controller so that the open-loop function of the controlled system has large amplitude in low frequencies. For robust stability, we want the open-loop function to have small magnitude in high frequencies. In general, it is reasonable that the function has a slope of -1 in the crossover region and at least a roll-off of 2 . Based on a great number of simulation experiments we select an open-loop function as follows:

$G_{\mathrm{d}}(s)=\frac{0.03}{s(s / 0.04+1)(s / 1.1+1)}$

A process control with robust stability can be achieved by applying the $H_{\infty}$ loop shaping procedure based on the nominal models with different choice of $N$.

Fig. 10 demonstrates the desired open-loop function $G_{\mathrm{d}}$ and the open-loop function $\widehat{G} K$ where $K$ is a synthesized controller based on a nominal model with $N=3$. The loop shaping procedure guarantees a $\widehat{G} K$ satisfying (27) and (28).

The left plot in Fig. 11 illustrates the robust stability of the controller synthesized using the desired open-loop function (35), and it shows that the robust stability criterion (34) holds. The weight function plotted with a solid line in the lower plot of Fig. 8 is used for robustness analysis.

To have some appreciation for how the controller would behave with the real process, it is tested using the frequency domain solution of the infinite dimensional model (1a) and (1b) as the process, 


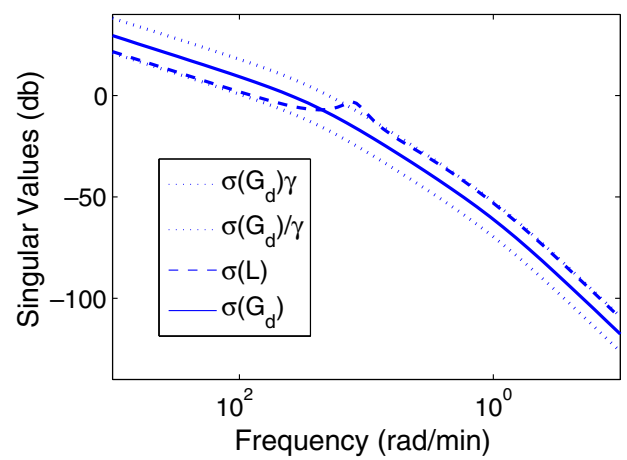

Fig. 10. Robust control synthesized using the loop shaping procedure based on a nominal model with $N=3$.

i.e. using the infinite dimensional transfer function (5) and (6), where the model is perturbed by taking different parameter values from the range shown in Table 1 . In the rest of this section, all the simulations of the perturbed process use the infinite dimensional transfer function as the simulation model. The upper plot in Fig. 12 demonstrates the nominal time response (solid line) and the perturbed time responses (dotted lines) of the controlled system, where there exist obvious oscillations caused by the parameter perturbation.

Next we test another controller synthesized using the following desired open-loop transfer function:

$G_{\mathrm{d}}(s)=\frac{0.03}{s(s / 0.02+1)(s / 1.1+1)}$

where a greater dominating time constant in $G_{\mathrm{d}}$ will make the reference tracking slightly slower but also allow increased stability margins. The right plot of Fig. 11 shows that the system is more robustly stable than the left plot, and the lower plot of Fig. 12 demonstrates better performance when subject to perturbations. The nominal performance is degraded in the sense of a slower time response.

A higher order nominal model usually has better accuracy than a lower order nominal model as can be seen in Fig. 8. Using a higher order model may help the robust control synthesis to achieve both high performance and good robustness. Thus we test an OC nominal model with $N=7$ in the controller synthesis, and select the open-loop function (35) in order to achieve a faster time response. The weight function for this nominal model is plotted using dot-dashed line in Fig. 8, and robustness analysis shows that the system is robustly stable. The upper plot in Fig. 13 shows perturbed step responses and illustrates the better performance compared to that obtained using the OC model with $N=3$.

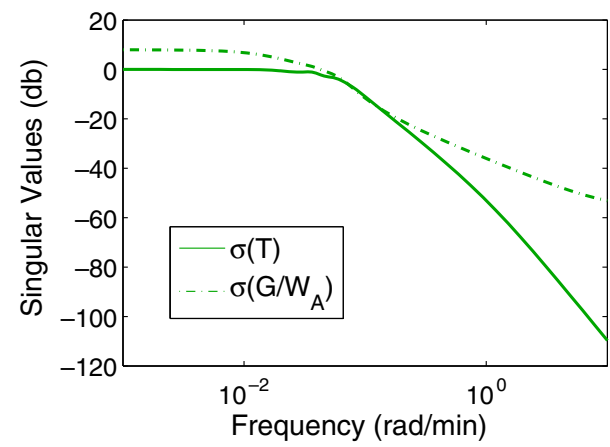

Now we test a controller synthesized based on the 17th order nominal model and compare the system performance with that using lower order models. The weight function defined for this high order model is shown in Fig. 8 using a dotted line, and the desired open-loop transfer function (35) is used. The lower plot in Fig. 13 illustrates the step response of the perturbed system, and there is no obvious difference between this plot and the upper one that shows the control system synthesized based on the nominal model with $N=7$.

In conclusion, using the nominal model with $N=3$, it is difficult to achieve good performance while tolerating parameter perturbations. If a slightly degraded performance can be accepted then the parameter perturbations are tolerated much better. Using a nominal model with a greater $N$, e.g. $N=7$, the system performance and robustness are obviously improved. However, the grade of improvement is not proportional to the increase of model order, and this can be seen when the nominal model with $N=17$ is chosen. Thus it may be unnecessary to choose a model with very high order. The low-order nominal models have advantages in controller implementation and computational time, and it is possible to select an appropriate low-order model by making a trade-off between simplicity of the model and robustness/performance of the closed-loop system.

\subsection{Application of the reduced models in state estimation}

A major motivation for using a physical process model is that the state $x$ then has a physical interpretation. Thus an estimate $\hat{x}$ of the state can provide valuable information about the current status of the process, in particular of quantities that cannot be measured directly.

The standard method of state estimation utilizes a state observer

$\dot{\hat{x}}=A \hat{x}+B u+K_{f}(y-C \hat{x}-D u)$

which requires a low-order process model of the form

$\dot{x}=A x+B u+M w_{1}$

$y=C x+D u+w_{2}$

where $w_{1}$ and $w_{2}$ are process disturbance and measurement disturbance, respectively. In order to show the potential of the reduced order model (10) for this purpose, a state observer is designed under the assumption that $w_{1}$ and $w_{2}$ are uncorrelated white noise signals with variance $R_{1}$ and $R_{2}$, respectively. For simplicity, it is also assumed that $w_{1}$ consists of $2(N+1)$ uncorrelated white noise signals with variance $r_{1}$, each affecting one state variable. Thus $R_{1}=r_{1} I_{2(N+1)}$ and $M=I_{2(N+1)}$. An identified process model with

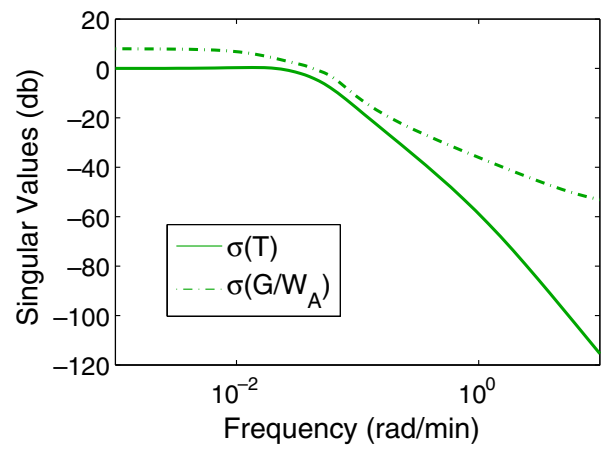

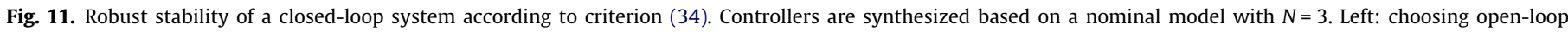
transfer function (35); right: choosing open-loop transfer function (36). 

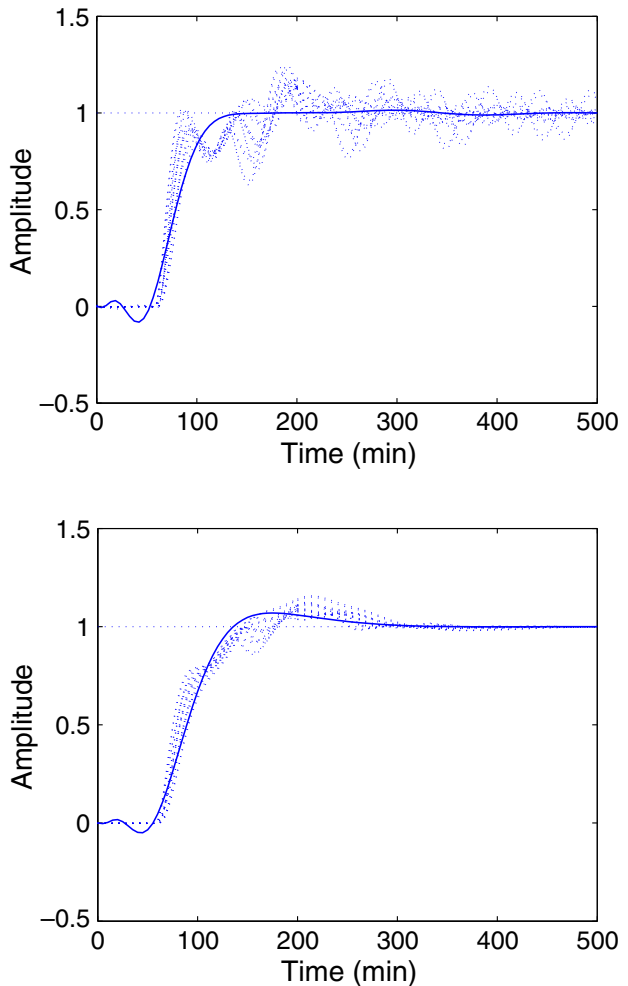

Fig. 12. Step responses of the nominal system (solid line) and the perturbed infinite dimensional system (dotted line). The controllers are synthesized based on a nominal model with $N=3$. Upper: choosing open-loop transfer function (35); lower: choosing open-loop transfer function (36).

$N=7$ is used in the experiments. A Kalman filter, i.e. (37) with optimal value of the observer feedback gain $K_{\mathrm{f}}$ is then obtained by solving a certain Riccati equation [17].

The Kalman filter is designed by manually adjusting the design parameters $r_{1}$ and $R_{2}$ to make the observer residual $r=y-C \hat{x}-D u$ resemble white noise for a set of measurement data of input $u$ and output $y$. The result is shown in Fig. 14 .

In order to determine the source of the estimation error, the observer is also applied to simulated output data $y$, generated using the frequency domain solution of (1), i.e. the frequency response $T_{\mathrm{f}}(z, \mathrm{i} \omega)$ for $z=1$. An estimated observer output $\hat{y}$ is then generated using the same observer gain $K_{\mathrm{f}}$ as above and the result is shown in Fig. 15. The model reduction is thus the only source of observer residual in this case and, since it is significantly smaller compared to Fig. 14, it is concluded that the major source of estimation error comes from imperfections in the process model (1), not the model reduction.

The state vector of the low-order model represents the temperatures in the collocation points $z=z_{0}, z_{1}, \ldots, z_{N+1}$. To obtain the temperatures in an arbitrary point $z$ one may use (13) and (14) to obtain the interpolation

$\hat{T}_{\mathrm{f}}(z, t)=C_{z} \hat{x}_{\mathrm{f}}(t)+D_{z} u(t)$

$\hat{T}_{\mathrm{c}}(z, t)=C_{z} \hat{x}_{\mathrm{c}}(t)+D_{z} u(t)$

where $C_{z}=\left[l_{1}(z) l_{2}(z) \cdots l_{N+1}(z)\right]$ and $D_{z}=l_{0}(z)$. The state $T_{\mathrm{f}}$ at two different positions ( $z=0.42$ and $z=1$ ), estimated using a process output generated by the frequency domain solution of (1) are shown in Fig. 16 (upper). It is compared to the process state calculated using the frequency domain solution of (1), i.e. the frequency response $T_{\mathrm{f}}(z, \mathrm{i} \omega)$. The agreement between estimated and calculated state
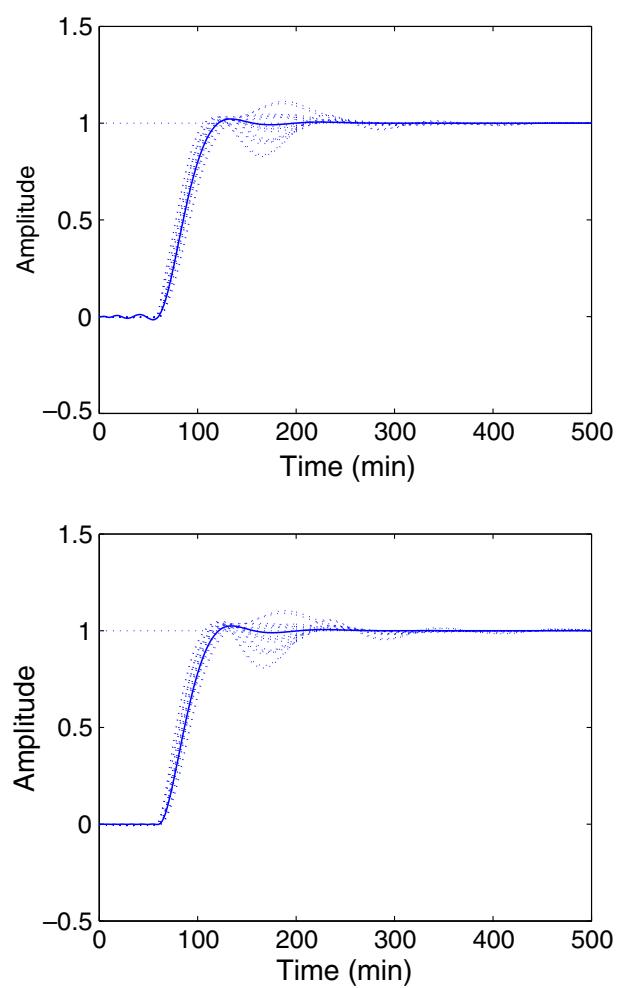

Fig. 13. Step responses of the nominal system (solid line) and the perturbed infinite dimensional system (dotted line). The desired open-loop transfer function is chosen as (35). Upper: the controller is synthesized based on a nominal model with $N=7$; lower: the controller is synthesized based on a nominal model with $N=17$.
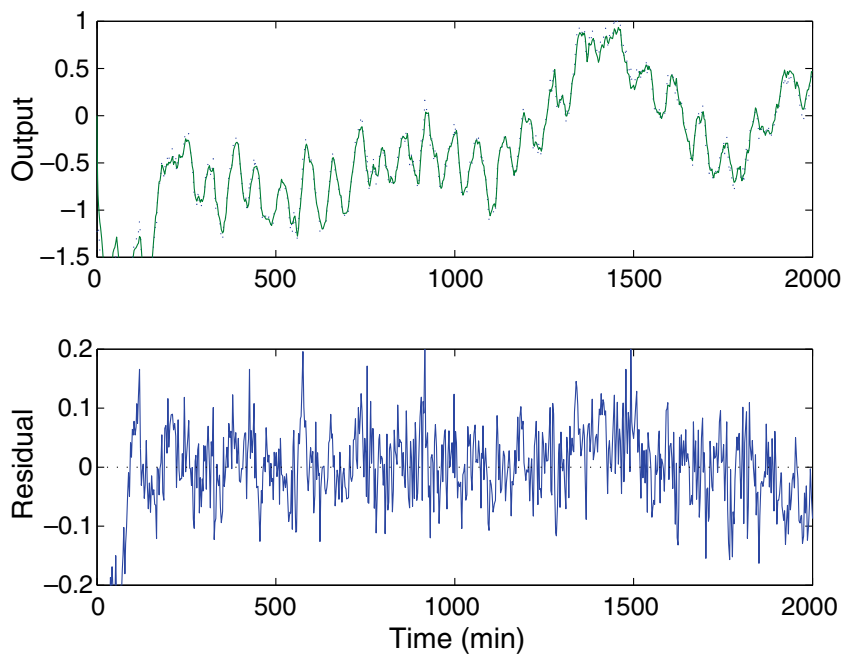

Fig. 14. Upper: Estimated (solid) and measured (dashed) process output. Lower: Observer residual.

shows that an observer for the reduced order process is capable of estimating the state of the PDE model (1).

To show that the observer can also estimate the state of the actual process, the $T_{\mathrm{f}}$ temperature at position $z=0.42$ is estimated using measurement data of the output. The estimate is compared to a measurement of the temperature at this position, denoted c6 in Fig. 1. The result is displayed in Fig. 16 (upper) and it is clear that they agree quite well. For reference, the simulated process state at the same position, calculated using the frequency domain solution of (1), is shown in the same plot. 

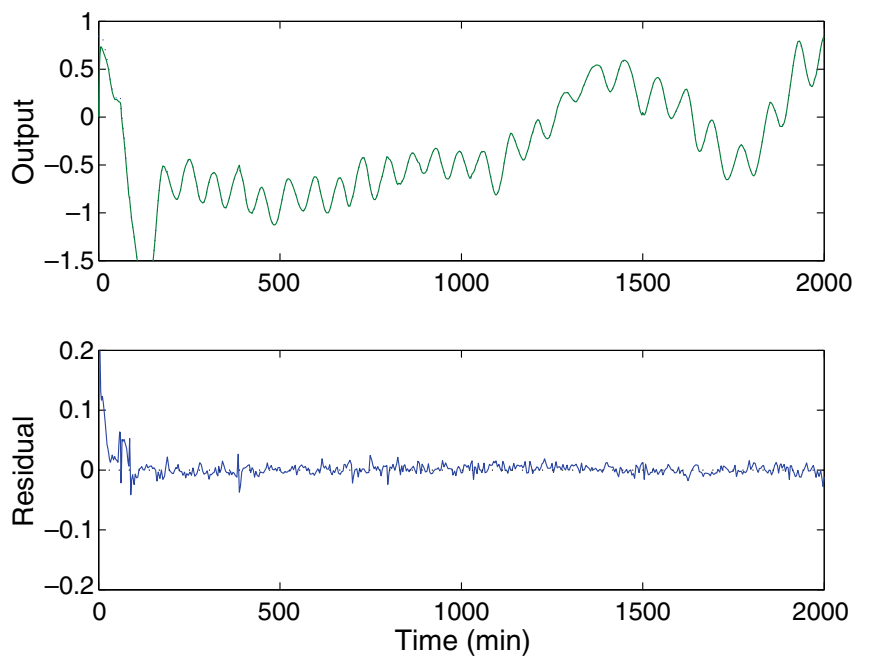

Fig. 15. Upper: Estimated process output (solid) and output generated from the frequency domain solution of (1) (dashed). Lower: Observer residual.
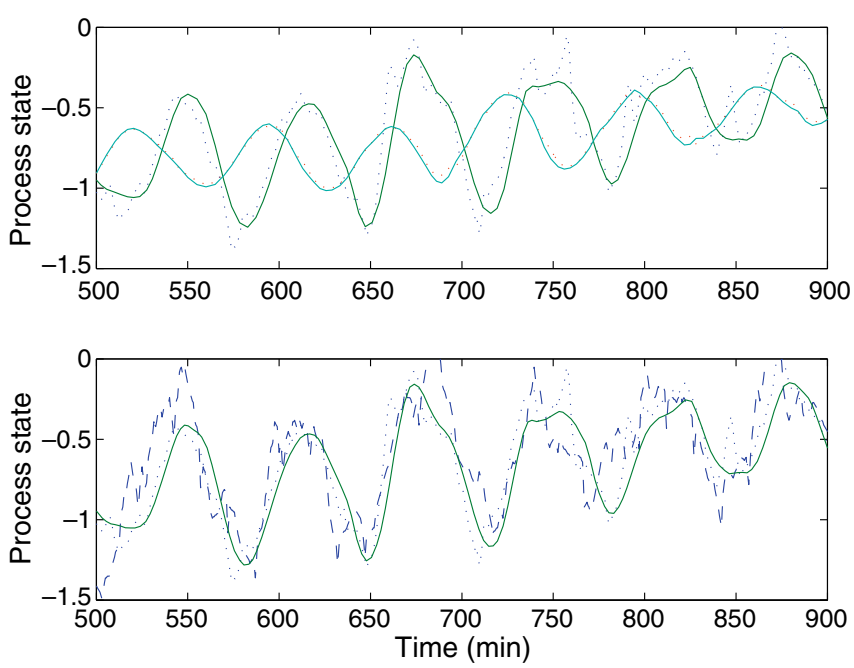

Fig. 16. Upper: Process state $T_{\mathrm{f}}$ at position $z=0.42$ and $z=0.7$ estimated using the output calculated from the frequency domain solution of (1) and compared to process state calculated from the frequency domain solution of (1) (dotted). Lower: Process state $T_{\mathrm{f}}$ at the $c 6$ position $(z=0.42)$, estimated (solid) using measured process output and compared to measured $c 6$ temperature (dashed) and simulated state (dotted) calculated from the frequency domain solution of (1).

\section{Conclusions}

The problem of applying DPS models for robust control and state estimation is addressed. By integrating model reduction with parameter identification and model uncertainty analysis, it is demonstrated how an appropriate trade-off between complexity and robust performance can be achieved. Measurement data from a continuous paper pulp digester is used throughout the study.

Low-order model approximation obtained using the OC approach is proposed to solve the problems due to the infinite dimensionality. The unknown parameters of the process model are identified based on the reduced models of different orders and the parameter estimates are shown to converge to small intervals with increasing model order.

The errors of the reduced models are estimated and analyzed using two different approaches. Comparing a low-order model to the infinite dimensional model with different parameter values ex- poses the errors mainly caused by parameter perturbation and model reduction. Estimating a model error model provides a picture of the overall errors including those caused by other reasons, such as model simplifications. By combining the results from the two approaches, a model error weight function used for robust control can be set up.

The loop shaping procedure is applied to synthesize $H_{\infty}$ optimal controllers. Simulation experiments demonstrate that it is possible to select an appropriate low-order model for controller synthesis by taking a trade-off between simplicity of the model and robustness/performance of the closed-loop system.

A Kalman filter is designed using a reduced process model in order to produce estimates of the process state. By comparing with measurement data it is demonstrated that the Kalman filter provides reliable estimates of the state at an arbitrary spatial point. Such an observer may be used for process monitoring and fault detection.

\section{Acknowledgements}

The authors would like to thank the Hjalmar Lundbohm Research Center for financial support. Measurement data from the Husum paper plant provided by M-real are greatly appreciated.

\section{References}

[1] P. Christofides, Control of nonlinear distributed process systems: recent developments and challenges, American Institute of Chemical Engineering Journal 47 (3) (2001) 514-518.

[2] P.D. Christofides, Special volume on 'control of distributed parameter systems', Computers and Chemical Engineering (2002).

[3] K.A. Hoo, D. Zheng, Low-order control-relevant models for a class of distributed parameter systems, Chemical Engineering Science 56 (2001) 6683-6710.

[4] K. Alhumaizi, R. Henda, M. Soliman, Numerical analysis of a reactiondiffusion-convection system, Computers and Chemical Engineering 27 (4) (2003) 579-594.

[5] M. Li, P.D. Christofides, Optimal control of diffusion-convection-reaction processes using reduced-order models, Computers and Chemical Engineering (2007), doi:10.1016/j.compchemeng.2007.10.018.

[6] T.T. Lee, F.Y. Wang, R.B. Newell, Robust model-order reduction of complex biological process, Journal of Process Control 13 (2002) 807-821.

[7] D. Zheng, K.A. Hoo, System identification and model-based control for distributed parameter systems, Computers and Chemical Engineering 28 (8) (2004) 1361-1375.

[8] T. Damak, Procedure for asymptotic state and parameter estimation of nonlinear distributed parameter bioreactors, Applied Mathematical Modelling 31 (2007) 1293-1307.

[9] A. Goyal, S. Mandapuram, B. Michniak, L. Simon, Application of orthogonal collocation and regression techniques for recovering parameters of a twopathway transdermal drug-delivery model, Computers and Chemical Engineering 31 (3) (2007) 107-120.

[10] A.J. Helmicki, C.A. Jacobson, C.N. Nett, Control oriented system identification: a worst-case/deterministic approach in $H_{\infty}$, IEEE Transactions on Automatic Control 36 (10) (1991) 1163-1176.

[11] M. Sznaier, M.C. Mazzaro, An LMI approach to control-oriented identification and model (in) validation of LPV systems, IEEE Transactions on Automatic Control 48 (9) (2003) 1619-1624.

[12] B.P. Rasmussen, A.G. Alleyne, A.B. Musser, Model-driven system identification of transcritical vapor compression systems, IEEE Transactions on Control Systems Technology 13 (3) (2005) 444-451.

[13] P.D. Christofides, Nonlinear and Robust Control of Partial Differential Equation Systems: Methods and Applications to Transport-Reaction Processes, Birkhauser, Boston, MA, 2001.

14] L. Ljung, Model validation and model error modeling, in: B. Wittenmark, A Rantzer, (Eds.), Proceedings of the Åström Symposium on Control, Studentlitteratur, Lund, Sweden, 1999, pp. 15-42.

[15] W. Reinelt, A. Garulli, L. Ljung, Comparing different approaches to model error modeling in robust identification, Automatica 38 (5) (2002) 787-803.

[16] K. Glover, D. McFarlane, Robust stabilization of normalized coprime factor plant descriptions with $H_{\infty}$-bounded uncertainty, IEEE Transactions on Automatic Control 34 (8) (1989) 821-830.

[17] S. Skogestad, I. Postlethwaite, Multivariable Feedback Control - Analysis and Design, John Wiley \& Sons, 1996.

[18] L. Ding, T. Gustafsson, A. Johansson, Model parameter estimation of simplified linear models for a continuous paper pulp digester, Journal of Process Control 17 (2007) 115-127.

[19] www.m-real.com. 
[20] J. Funkquist, Modeling and identification of a distributed parameter process: the continuous digester. Ph.D. Thesis, Automatic Control, Department of Signals, Sensors and Systems, Royal Institute of Technology, Sweden, 1995.

[21] LennartLjung. Ljung, System Identification. Theory for the User, second ed., Prentice Hall, Upper Saddle River, NJ, USA, 1999.

[22] MathWorks. System identification toolbox.

[23] J. Sjöberg, Q.H. Zhang, L. Ljung, Nonlinear black-box modeling in system identification: a unified overview, Automatica 31 (12) (1995) 1691-1724.
[24] S. Bhartiya, P. Dufour, F.J. Doyle III, Fundamental thermal-hydraulic pulp digester model with grade transition, AIChE Journal 49 (2) (2003) 411425.

[25] MathWorks. Robust control toolbox.

[26] D.C.McFarlane, McFarlane, K.Glover, Glover, Robust Controller Design using Normalised Coprime Factor Plant Descriptions, Lecture Notes in Control and Information Sciences, Springer-Verlag, 1989. 